\title{
Colchicine for steroid-resistant recurrent pericarditis in a child
}

\author{
Ju Hee Shin, Dong Hyun Lee, Hee Joung Choi \\ Department of Pediatrics, Keimyung University School of Medicine, Daegu, Korea
}

Recurrent pericarditis is rare in children and is considered idiopathic in most cases. Its course is chronic, and preventing recurrences is important for the patient's quality of life. Although a treatment strategy in pediatric recurrent pericarditis has not yet been established, non-steroidal anti-inflammatory drugs (NSAIDs) are the most common treatment for management of this condition, followed by corticosteroids, colchicine, immunosuppressive agents, immunoglobulins, and interleukin- $1 \beta$ receptor antagonists (e.g. anakinra). Herein, we report a case of recurrent pericarditis with pericardial effusion in a 5-year-old child who presented with fever and epigastric pain. He responded poorly to NSAIDs and corticosteroid therapy, but was successfully treated with colchicine.

Keywords: Children; Colchicine; Recurrent pericarditis

\section{INTRODUCTION}

Pericarditis is a condition caused by inflammation of the pericardium that characterized by typical chest pain, pericardial friction rub, electrocardiographic changes, and pericardial effusion [1]. Recurrent pericarditis is defined as the return of symptoms and signs of pericarditis after a symptom-free interval of 4-6 weeks [2]. The recurrence rate of pericarditis in children is similar to that in adults, occurring in up to $15-40 \%$ of cases $[3,4]$. The prognosis of recurrent pericarditis is generally favorable, however, patients who present with fever, subacute symptomatology, large pericardial effusion, and/or cardiac tamponade, and are non-responsive to non-steroidal anti-inflammatory drugs (NSAIDs) tend to have a poorer prognosis [5].

Although the standard treatment strategy in the pediatric

Received: December 19, 2017, Revised: January 4, 2018 Accepted: January 17, 2018

Corresponding Author: Hee Joung Choi, Department of Pediatrics, Keimyung University School of Medicine, 56, Dalseong-ro, Jung-gu, Daegu 41931, Korea

Tel: +82-53-250-7524, Fax: +82-53-250-7783

E-mail: joung756@dsmc.or.kr population has not yet been established, several effective agents are used to treat recurrent pericarditis including NSAIDs, corticosteroids, colchicine, immunosuppressive agents, and immunoglobulins [4]. NSAIDs plus colchicine is considered to be first-line therapy in adult patients with recurrent pericarditis; however, in the pediatric population, corticosteroids are used more commonly than colchicines [6]. Here, we present the case of a 5-year-old child with recurrent pericarditis and pericardial effusion that was caused by coxsackievirus, and was non-responsive to both NSAIDs and steroid therapy, but was treated effectively with colchicine therapy.

\section{CASE}

A 5-year-old previously healthy boy presented to the emergency department with a 6-day history of fever (up to $38.7^{\circ} \mathrm{C}$ ) and a 2-day history of epigastric pain. Upon general examination, he appeared acutely ill, with initial vital signs as follows: blood pressure of 100/54 mmHg, heart rate of 92 beats/min, respiratory rate of 24 breaths/min, and body temperature of $38.0^{\circ} \mathrm{C}$. A diffuse erythematous rash covered his whole body, which had been present for 7 days. Initial blood tests revealed the following results: erythrocyte sedimentation rate 114

Copyright $($ C) 2018 Yeungnam University College of Medicine

This is an Open Access article distributed under the terms of the Creative Commons Attribution Non-Commercial License (http://creativecommons.org/licenses/by-nc/4.0/) which permits unrestricted non-commercial use, distribution, and reproduction in any medium, provided the original work is properly cited. 

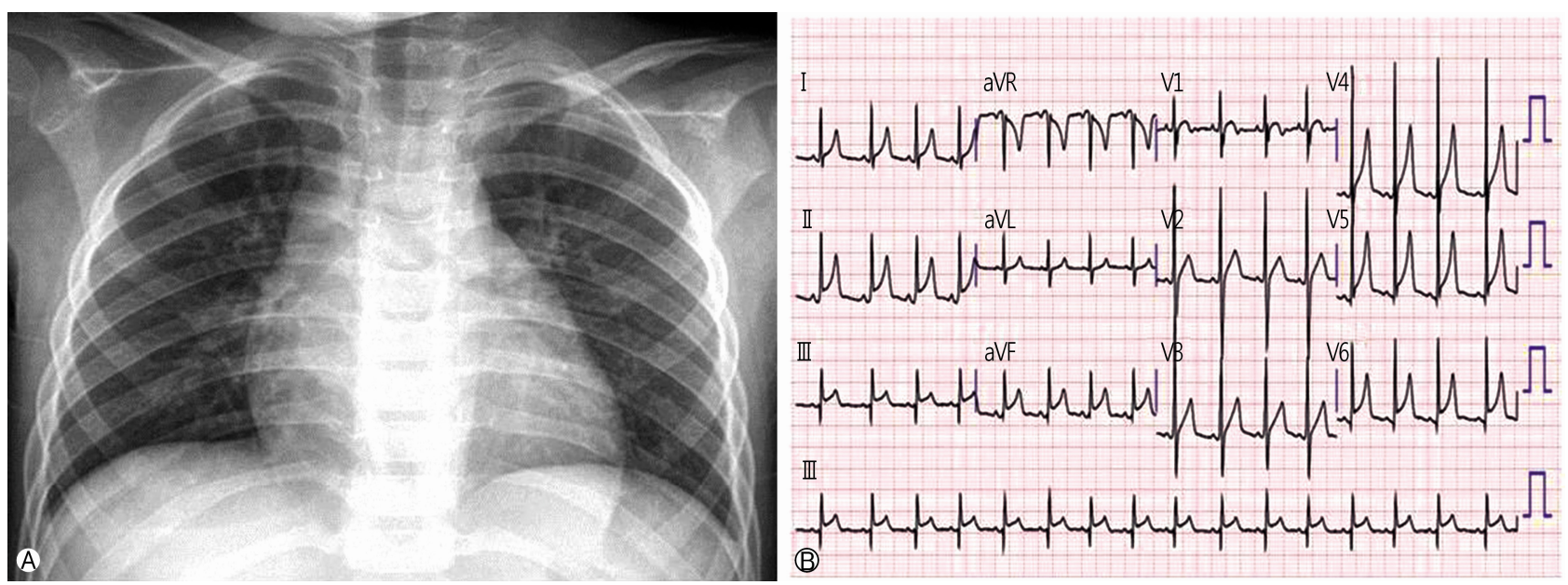

Fig. 1. Chest radiograph and electrocardiography of the patient on admission day. (A) Chest radiograph showed cardiomegaly with a cardiac-thoracic ratio of 0.56. (B) Electrocardiography showed ST elevation was observed in leads II, III, aVF, and V4-V6.

$\mathrm{mm} / \mathrm{hr}$, C-reactive protein (CRP) $15.7 \mathrm{mg} / \mathrm{dL}$, N-terminal proB-type natriuretic peptide (NT-proBNP) $1,573 \mathrm{pg} / \mathrm{mL}$, creatinine kinase (CK)-MB $<0.5 \mathrm{ng} / \mathrm{mL}$ and troponin I $0.022 \mathrm{ng} / \mathrm{mL}$. Chest radiograph revealed cardiomegaly with a cardiac-thoracic (CT) ratio of 0.56 (Fig. 1A), while electrocardiogram (ECG) showed a concave ST-segment elevation in leads II, III, aVF, and V4-V6 (Fig. 1B). A transthoracic echocardiogram revealed normal systolic and diastolic function of the left ventricle, no pericardial effusion was observed, and the coronary artery system was normal in size. Although there were significant ECG abnormalities, typical chest pain and a pericardial effusion were not present. The patient was treated with the standard dose of ibuprofen $(1.5 \mathrm{mg} / \mathrm{kg} /$ day $)$ for fever, and he received systemic antibiotics for the possibility of a bacterial cause, as well as intravenous immunoglobulin (IVIG) for the possibility of a viral cause of his condition.

On the third hospital day, the patient remained febrile and a follow-up echocardiogram showed evidence of minimal pericardial effusion; therefore, we increased the dosage of ibuprofen (30 mg/kg/day) and administered aspirin (60 mg/kg/day) to treat acute pericarditis. Despite treatment with ibuprofen and aspirin, the patient remained febrile and his condition did not improve. Serial echocardiograms revealed an increasing pericardial effusion, reaching up to $10 \mathrm{~mm}$ on the sixth hospital day (Figs. 2A, 2B). Chest radiograph showed increased cardiomegaly with a CT ratio of 0.69 (Fig. 2C), and an ECG showed normal ST segments with $\mathrm{T}$ wave flattening (Fig. 2D). We performed a pericardiostomy and $100 \mathrm{~mL}$ of yellowish serous fluid was aspirated. Analysis of the peri- cardial fluid revealed a white blood cell count of $1,080 / \mathrm{mm}^{3}$, consisting of $65 \%$ polymorphonuclear leukocytes and $35 \%$ lymphocytes, as well as an adenosine deaminase level of 41.7 IU/L. Acid-fast bacilli stain, and gram stain and culture of the pericardial fluid were negative. Following the pericardiostomy, glucocorticoid therapy with prednisolone at $1 \mathrm{mg} / \mathrm{kg} /$ day was prescribed, and the patient's fever subsided by the next day.

On the fifteenth hospital day, the patient was discharged in good condition with no pericardial effusion, and prednisolone treatment was continued. The viral serology result was positive for coxsackievirus B1, while other tests for bacterial and viral serologies, tuberculosis, and collagen-vascular disease were all negative. In the out-patient department (OPD), prednisolone was tapered by one half dose each week, and was ultimately discontinued at 4 weeks after hospital discharge.

Nine weeks after hospital discharge (5 weeks after discontinuation of prednisolone), the patient was readmitted due to a history of fever and epigastric pain for 1 day, symptoms that were similar to his previous admission. Laboratory test results revealed a CRP level of $11.6 \mathrm{mg} / \mathrm{dL}$ and an elevated NT-proBNP level of $1,884 \mathrm{pg} / \mathrm{mL}$. A chest radiograph revealed cardiomegaly with a CT ratio of 0.67 , an ECG revealed ST-segment elevation, and an echocardiogram showed pericardial effusion $(9.3 \mathrm{~mm})$. He was treated with ibuprofen (30 $\mathrm{mg} / \mathrm{kg} /$ day $)$ and prednisolone $(2 \mathrm{mg} / \mathrm{kg} /$ day $)$ for recurrent pericarditis. Fever and pain subsided after 1 day of medication, and he was discharged on the fifth hospital day with a nor- 

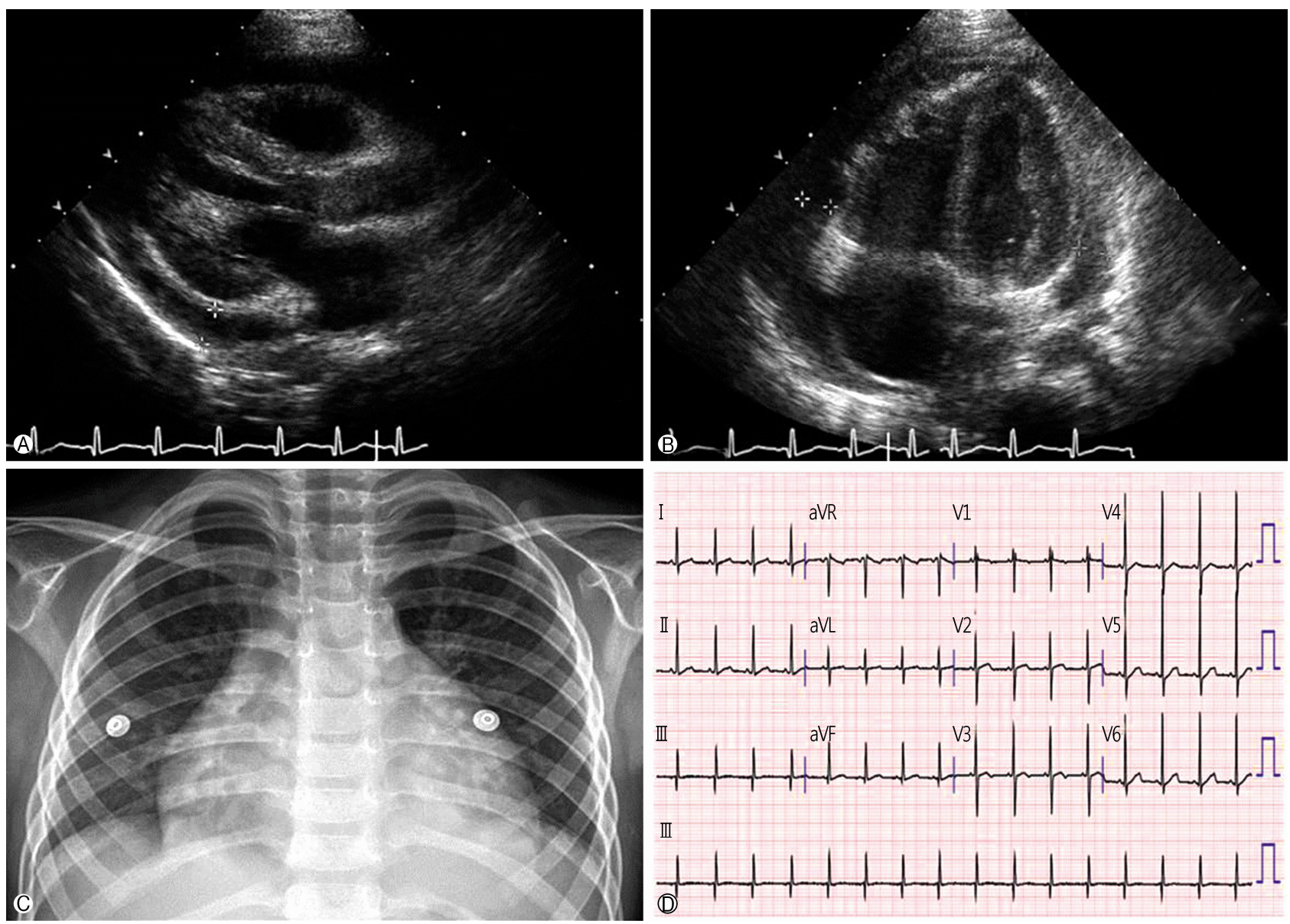

Fig. 2. Imaging studies of the patients on the sixth hospital day. (A) Pericardial effusion approximately $10 \mathrm{~mm}$ on the posterior side of the left ventricle in parasternal long axis view of Echocardiogram. (B) Pericardial effusion approximately $8.3 \mathrm{~mm}$ on the right side and $8.5 \mathrm{~mm}$ on the left side of the heart in apical four chamber view of Echocardiogram. (C) Chest radiograph showed cardiomegaly with a cardiac-thoracic ratio of 0.69. (D) Electrocardiography showed normal ST segment and T wave flattening.

malized CRP level $(0.94 \mathrm{mg} / \mathrm{dL})$ and no pericardial effusion. Laboratory tests including bacterial and viral serologies were performed and there was no positive results.

After discharge, prednisolone was subsequently tapered by a half dose every 2 weeks for 2 months. While tapering the prednisolone, the patient developed fever and epigastric pain again. His CRP level was $14.49 \mathrm{mg} / \mathrm{dL}$ and his NT-proBNP level was $285 \mathrm{pg} / \mathrm{mL}$. There was also cardiomegaly, with a CT ratio of 0.56 , observed on a chest radiograph, and pericardial effusion (4.5-5.5 mm) visualized on an echocardiogram. Colchicine therapy $(1 \mathrm{mg} /$ day $)$ was initiated for the recurrence of pericarditis, and was given in addition to ibuprofen (30 $\mathrm{mg} / \mathrm{kg} /$ day). The fever and chest pain disappeared 3 days after the initiation of therapy, and he was discharged on the ninth hospital day with a decreased CRP level $(1.68 \mathrm{mg} / \mathrm{dL})$ and complete resolution of the pericardial effusion. Laboratory tests including bacterial and viral serologies were repeated and there were no positive results.

In the OPD, colchicine was continued at a dose of $1 \mathrm{mg} /$ day for 6 months, then tapered at a dose of $0.5 \mathrm{mg} /$ day for 6 months with follow-up chest radiography and echocardiogram. There was no recurrence of pericarditis after discontinuation of the colchicine. At 30 months following the end of treatment, he remained in good physical condition, with no clinical symptoms of recurrence of pericarditis.

\section{DISCUSSION}

Chest pain is the presenting symptom in almost all adult patients with pericarditis [1]. However, in pediatric patients, chest pain may not be a clear presenting symptom because younger children cannot precisely describe typical chest pain 
[6. Systemic signs and symptoms such as fever, fatigue, nausea or vomiting, pleuritis, and pneumonia, are more commonly associated with pediatric patients than adults [6. In this case, the patient did not complain of typical chest pain, but he initially presented with fever, rash and abdominal pain. In general, appropriate and timely therapy can lead to a rapid recovery from acute pericarditis. However, the patient did not respond well to the first course of treatment with NSAIDs (ibuprofen and aspirin), possibly because the treatment was delayed until the development of pericardial effusion.

The known causes of recurrent pericarditis are viral infections, post-pericardial injury syndromes, autoinflammatory diseases, neoplastic pericardial diseases, and genetic disease [2,7]. However, most cases remain idiopathic, and it has been suggested that autoimmune mechanisms may be the main precursors to the development of idiopathic recurrent pericarditis [4]. Inadequate treatment regimens, such as insufficient dosage or duration of NSAID therapy, or the tapering of corticosteroids too soon, may represent the other primary cause of recurrence [2,4]. In short, proper dosing and duration of treatment is important to prevent recurrence.

NSAIDs are a commonly used first-line treatment for acute and recurrent pericarditis in both adult and pediatric patients $[4,8]$. Commonly used drugs include aspirin (70-100 mg/kg/ day), ibuprofen (30-40 mg/kg/day), and indomethacin (1-2 $\mathrm{mg} / \mathrm{kg} /$ day) administrated until complete resolution of symptoms and normalization of CRP are observed [4].

Colchicine is also considered first-line treatment for both acute and recurrent pericarditis in adults [2,8]. Although it is not yet routinely used in children, a recent study of pediatric recurrent pericarditis reported that colchicine, when added to NSAID therapy, reduced the recurrence rate by approximately 50\% [3,6. According to the European Society of Cardiology (ESC) guidelines, colchicine is considered an effective adjunctive drug to NSAIDs, rather than corticosteroids [8]. Even though colchicine is well tolerated in children, the evidence regarding its effects in children is still scarce and contradictory [9]. The recommended dose is $0.5 \mathrm{mg} /$ day for children less 5 years of age and $1.0-1.5 \mathrm{mg} /$ day those over 5 years old, and while recommended duration is 3 months for acute pericarditis and 6-12 months for recurrent pericarditis [10]. Colchicine has few serious side effects including gastrointestinal symptoms with anorexia, renal impairment, alopecia, hepatotoxicity, and myelosuppression [10]. In our case, the first recurrence developed 5 weeks after discontinuation of prednisolone, and the second recurrence developed during the tapering of prednisolone. The trigger for recurrence might be rapid tapering of the steroids. Notably, our patient did not have any further recurrence after colchicine treatment, which he completed without any side effects.

Corticosteroids are used when the patient does not respond to NSAIDs, or has had severe symptoms [7]. However, the long-term use of steroids carries the risk of complications such as detrimental effects on bone metabolism (osteoporosis) and growth, and recurrence of pericarditis, which occurs more frequently in patients treated with steroids than in those treated with colchicines [6]. Therefore, steroids are considered to be a second-choice therapy in recurrent pericarditis and their use is limited to patients who are refractory to first-line treatment, or when a contraindication to NSAIDs and colchicine is present [7]. However, in pediatric patients with recurrent pericarditis, steroids are more commonly used than colchicine when compared to adult patients [6]. When glucocorticoids are required, low dose therapy $(0.2-0.5 \mathrm{mg} / \mathrm{kg} /$ day $)$ is preferable to higher doses ( $1 \mathrm{mg} / \mathrm{kg} /$ day $)$, and they should be tapered very slowly, for example at 1-2 weeks intervals over a period of 2-4 months [1,11]. During the tapering of steroids, adding colchicine may be considered to prevent recurrence [12]. In our case, the high dose (1 mg/kg/day) and somewhat large dosage decrement of corticosteroids may have triggered the recurrence. If a patient does not respond to NSAIDs, colchicine or low dose corticosteroids, and requires long-term high-dose corticosteroids, their condition is referred as refractory pericarditis [2]. In such cases, immunosuppressants such as azathioprine, methotrexate, cyclophosphamide, cyclosporins, IVIG, and interleukin- $1 \beta$ receptor antagonists (anakinra) can be used [4,13,14].

In conclusion, we report here the case of a 5-year-old boy with recurrent pericarditis who had two episodes of recurrence despite NSAID and corticosteroid therapy, but was then successfully treated with colchicine without side effects. The course of recurrent pericarditis is somewhat long and unpredictable, but the activity of the disease often decreases gradually. Proper management of recurrences can improve the quality of life. As in our case, colchicine might be effective at preventing recurrences in children with recurrent pericarditis. 


\section{CONFLICT OF INTEREST}

No potential conflict of interest relevant to this article was reported.

\section{ORCID}

Juhee Shin, https://orcid.org/0000-0002-0121-1825

Hee Jung Choi, https://orcid.org/0000-0002-7119-4194

\section{REFERENCES}

1. LeWinter MM. Clinical practice. Acute pericarditis. N Engl J Med 2014;371:2410-6.

2. Imazio M, Gribaudo E, Gaita F. Recurrent pericarditis. Prog Cardiovasc Dis 2017;59:360-8.

3. Shakti D, Hehn R, Gauvreau K, Sundel RP, Newburger JW. Idiopathic pericarditis and pericardial effusion in children: contemporary epidemiology and management. J Am Heart Assoc 2014;3:e01483.

4. Gaspari S, Marsili M, Imazio M, Brucato A, Di Blasi Lo Cuccio C, Chiarelli F, et al. New insights in the pathogenesis and therapy of idiopathic recurrent pericarditis in children. Clin Exp Rheumatol 2013;31:788-94.

5. Imazio M, Spodick DH, Brucato A, Trinchero R, Adler Y. Controversial issues in the management of pericardial diseases. Circulation 2010;121:916-28.

6. Imazio M, Brucato A, Pluymaekers N, Breda L, Calabri G,
Cantarini L, et al. Recurrent pericarditis in children and adolescents: a multicentre cohort study. J Cardiovasc Med (Hagerstown) 2016;17:707-12.

7. Raatikka M, Pelkonen PM, Karjalainen J, Jokinen EV. Recurrent pericarditis in children and adolescents: report of 15 cases. J Am Coll Cardiol 2003;42:759-64.

8. Adler Y, Charron P, Imazio M, Badano L, Barón-Esquivias G, Bogaert J, et al. 2015 ESC Guidelines for the diagnosis and management of pericardial diseases. Eur Heart J 2015; 36:2921-64.

9. Alabed S, Pérez-Gaxiola G, Burls A. Colchicine for children with pericarditis: systematic review of clinical studies. Arch Dis Child 2016;101:953-6.

10. Imazio M, Adler Y. Treatment with aspirin, NSAID, corticosteroids, and colchicine in acute and recurrent pericarditis. Heart Fail Rev 2013;18:355-60.

11. Imazio M, Brucato A, Cumetti D, Brambilla G, Demichelis B, Ferro S, et al. Corticosteroids for recurrent pericarditis: high versus low doses: a nonrandomized observation. Circulation 2008;118:667-71.

12. Artom G, Koren-Morag N, Spodick DH, Brucato A, Guindo J, Bayes-de-Luna A, et al. Pretreatment with corticosteroids attenuates the efficacy of colchicine in preventing recurrent pericarditis: a multi-centre all-case analysis. Eur Heart J 2005; 26:723-7.

13. del Fresno MR, Peralta JE, Granados MÁ, Enríquez E, Domínguez-Pinilla N, de Inocencio J. Intravenous immunoglobulin therapy for refractory recurrent pericarditis. Pediatrics 2014; 134:e1441-6.

14. Scardapane A, Brucato A, Chiarelli F, Breda L. Efficacy of an interleukin-1 $\beta$ receptor antagonist (anakinra) in idiopathic recurrent pericarditis. Pediatr Cardiol 2013;34:1989-91. 